\title{
NUMERICAL AND EXPERIMENTAL GENERATION OF FREAK WAVES
}

\author{
Nick Markov, Grigor Nikolov and Rumen Kishev* \\ Institute of Metal Science, Equipment and Technologies \\ with Hydro- and Aerodynamics Centre "Acad. Angel Balevski", \\ Bulgarian Academy of Sciences \\ 1, William Froude St., 9003 Varna, Bulgaria, \\ e-mails:n.markov@bshc.bg; grigor.plamenov@gmail.com; \\ e-mail: r.kishev@bshc.bg
}

\begin{abstract}
Control signals with simultaneous modulation of periods and amplitudes were fine-tuned and fed to a wave flap for a generation of freak waves. The meshless Smoothed Particle Hydrodynamics method was used to predict the location and the amplitude of the maximum wave crest. The time series output from the DualSPHysics software was validated experimentally in the seakeeping wave basin at the Bulgarian Ship Hydrodynamics Centre. The experimental data and the simulations are in good agreement.

Keywords: freak wave, rogue wave, smoothed particle hydrodynamics, basin tests, experimental validation.
\end{abstract}

\section{INTRODUCTION}

Climate change, expressed in an increase in average temperatures, an increase in the intensity of atmospheric phenomena, the melting of polar ice and the associated expected rise in sea level, is considered by the European Commission and the world scientific community as a likely scenario. It is causing a global change in our way of life, and in particular to the industrialization and urbanization of the offshore environment. There will be an increasing need to transfer accommodations and production activities offshore. To minimize the risk to human life in the new habitat, it is necessary to study and take into account the changing operating conditions and the appearance of hitherto considered abnormal and rare phenomena, such as extremely high waves.

\footnotetext{
* Corresponding author.

DOI: 10.7546/EngSci.LVIII.21.02.06
} 
The phenomenon of the appearance of abnormal waves is mainly studied numerically within the framework of theoretical scope of oceanography. However, the interaction of these waves, also known as "killer waves" or "freak waves", with obstacles and in particular with marine facilities, has been studied to a much lesser extent. The theoretical studies have been limited to qualitative results in this case.

To validate the numerical simulations, it would be necessary to model the abnormal waves in experimental wave basins and to measure the impact on different marine structures.

The freak wave represents a single extreme event, statistically considered a rare random peak in a general sequence of ordinary wind waves [1]. The wind waves normally occurring in the ocean have a relatively small slope, i.e. their wave heights are much smaller than their wavelengths. While the freak wave is a collapsing wave that has an unusually steep slope and a very shallow trough. The first technically measured and recorded abnormal wave in natural conditions was the so-called "New Year's wave" with a height equal to 25.6 $\mathrm{m}$ [2]. According to some sources, the height of an abnormal wave can reach even higher values [3].

The abnormal wave cannot be described by the spectral theory of progressive waves. There is still no generally accepted definition of the phenomenon, but according to the criterion common in the scientific literature, the Hmax/Hs ratio of the maximum height (from trough to crest) to the significant wave height must exceed 2.4, which is more than eight times the standard deviation [3].

The height of the wave is limited by the wave breaking phenomena. The wave shape formed before braking is similar to a wind wave with extreme steepness [4]. The killer wave profile determines the force exerted on ships and marine equipment [5-6]. The hydrodynamic impact during the collapse of a killer wave crest can produce cracks and leaks. A rollover with fatal consequences is possible in cases where a ship is located at an unfavorable angle to the wave. The abnormal waves also occur suddenly and do not allow the vessels to maneuver to avoid them.

Numerous observations, measurements and analysis of field data, as well as the theories describing the abnormal waves origin, identify the following major factors contributing to the freak wave occurrence: currents, bottom topography, wind and wave fields instability, spatial-dispersion focusing, and wave modulation. The degree of nonlinearity depends on the wave steepness and represents another important factor in the formation of an abnormal wave. Solitons (single amplitude single waves) are considered prototypes of killer

Engineering Sciences, LVIII, 2021, No. 2 
waves. Theories explaining the origin of the killer waves continue to evolve, with the application range of a given theory depending on the relation between the wave steepness and the water depth [7].

Due to the complexity of the mathematical models, physical modelling is currently the most promising research approach to estimate the slamming pressures exerted by freak waves on marine structures. Experimental facilities, such as the Technical University of Berlin [8-9], the Norwegian Institute of Technology Marintek [10] and the Dutch MARIN Institute, have already attempted to correctly model scaled-down abnormal waves in their wave basins, and to measure the wave impact on various structures.

Our study includes a new application of the meshless Smoothed-ParticleHydrodynamics method for numerical freak wave generation. The method predicts excellently the nonlinear maximum wave crest, which was validated experimentally with test measurements in a basin. The results of this study can further advance the design of fixed and floating facilities, reduce operating risks, improve metocean design basis and support the development of "blue" growth.

\section{FREAK WAVE GENERATION}

Wave phase velocity $(\lambda / T)$ depends on the wavelength $\lambda$ and the wave period $T$. Shorter waves propagate slower than longer waves. To produce an abnormally high wave crest we need to fine-tune a wave train, selecting variable wave periods so that the longer-period wave crests can reach the shorter waves at a certain point, and interfere constructively. To achieve that, we start the time series with shorter waves and gradually increase the wave period.

Figure 1 shows the time series fed to a $3 \mathrm{~m}$ tall rotating wave flap, used for wave generation at the Bulgarian Ship Hydrodynamics Centre (BSHC) seakeeping basin. The time series has three smoothly connected sections depicted in yellow and white regions. The desired wave modulation happens in the middle white section. The first yellow region includes a regular wave. Its purpose is to ramp up the energy in the basin. Without it, the modulated wave dissipates fast and does not produce an abnormal crest. The third yellow region simply flattens the signal to the wave-maker, which leaves room for further amplitude increases (if needed) without exceeding the operating range of the flap.

The modulated middle section is composed taking into account the capability range of the wave-maker and the steepness limit for wave breaking (the breaking is to be avoided since it would lower the maximum crests). To main- 
tain the steepness limit, the wave amplitudes need to be increased with the wave periods.

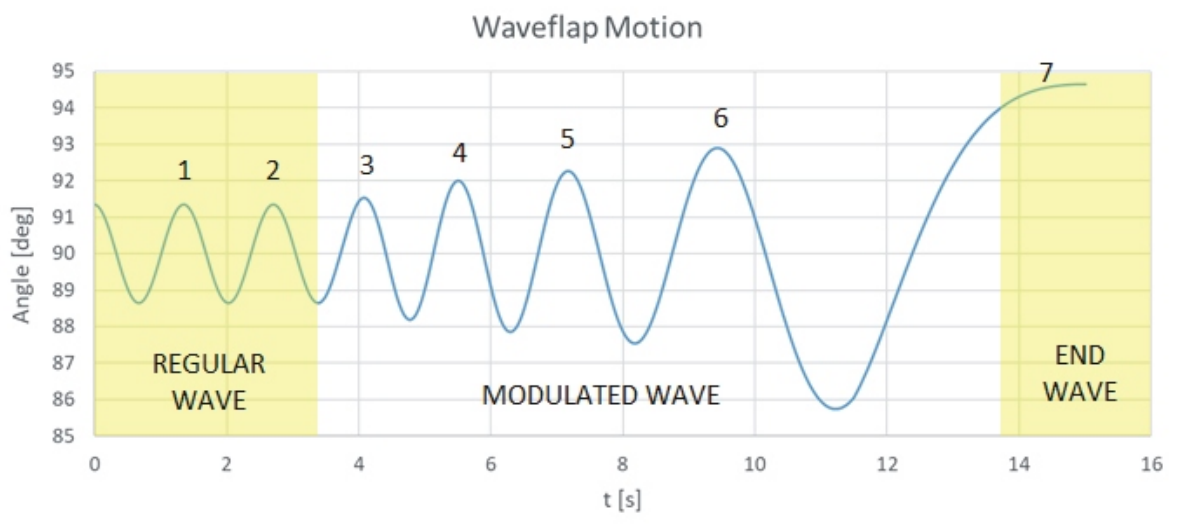

Fig. 1. The control signal to the wave maker

Figure 2 shows an approximately estimated location of the freak wave, which would be needed to place correctly the amplitude measurements in the numeric simulation. The estimate is based on phase velocities corresponding to average wave periods from the time series in Fig. 1. The average wavelengths

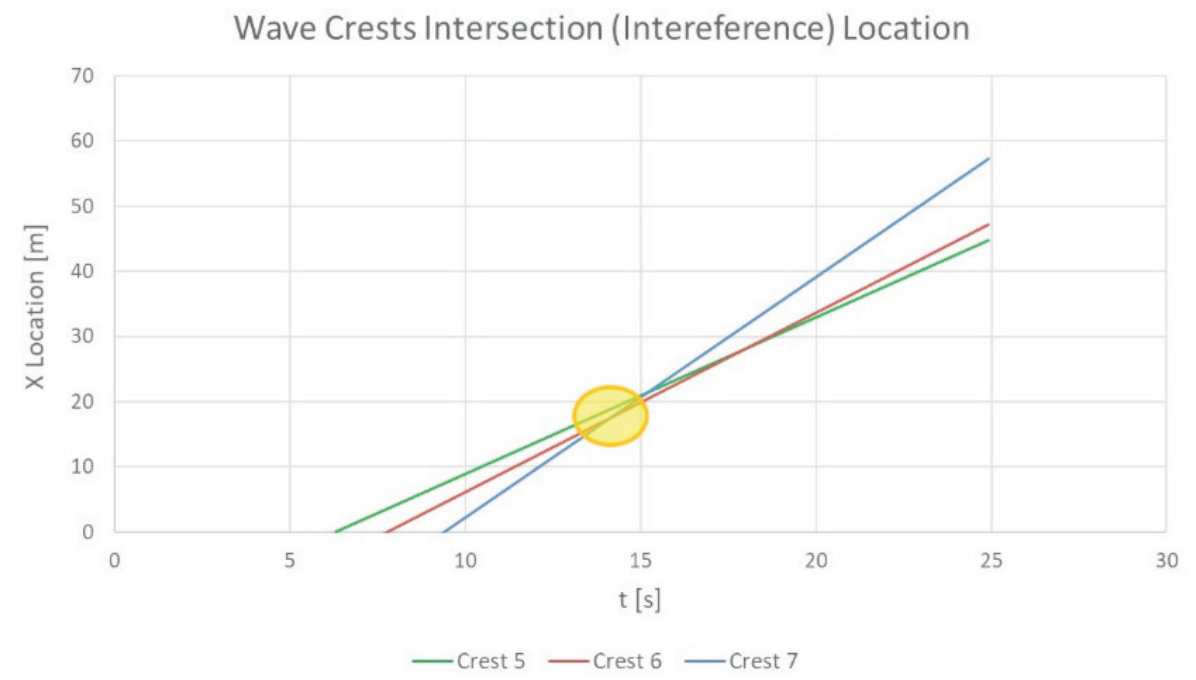

Fig. 2. Estimation of the freak wave location taking into account the three largest crests 
are also known from the standard calibration curves for the wavemaker. The estimate also takes into account the wavelength decrease $(<7 \%)$ due to shallow water effects, affecting the longest waves, according to the wave dispersion equation:

$$
\lambda=\frac{g T^{2}}{2 \pi} \tanh \left(2 \pi \frac{d}{\lambda}\right)
$$

where $d$ is the water depth, and $g$ is the gravitational acceleration. The location estimate is only approximate because of the gradual increase of the wave parameters, in our case. The next step is to get a better estimate for the freak wave amplitude and location using the meshless DualSPHysics solver.

\section{NUMERICAL SIMULATION}

DualSPHysics software is used to simulate freak waves in a seakeeping basin with dimensions $60 \times 40 \times 2.460 \mathrm{~m}$. The software is based on the Smoothed Particle Hydrodynamics (SPH) model named SPHysics - an open-source SPH code, inspired by the formulation in [11] and developed jointly by researchers at the Johns Hopkins University (U.S.A.), the University of Vigo (Spain), the University of Manchester (U.K.) and the University of Rome La Sapienza (Italy). The code was developed over the years primarily to study free-surface flow phenomena where Eulerian methods can be difficult to apply, such as waves, and the impact of dam-breaks on offshore structures.

The SPH method is meshless and discretizes the fluid into a set of particles, where the physical quantities (position, velocity, density, and pressure) are obtained as an interpolation of the corresponding quantities of the surrounding particles [12]. The weighted contribution of those particles is obtained using a kernel function $W a b$ with an area of influence that is defined using a characteristic smoothing length $h$. The Wendland kernel [13] is used in the DualSPHysics software and it is defined to vanish beyond $2 h$. The particles are initially created with an inter-particle distance $d p(=0.007 \mathrm{~m}$ in our case), which is used as a reference value to define the smoothing length using $h=2 d p$. The Navier-Stokes equations can be then written in a discrete SPH formalism using $W a b$ as the kernel function, which depends on the normalized distance between a given particle and its neighboring particles. Such discretization allows computing the position, velocity, and density of each SPH particle within the selected wave basin domain at every point in time.

The SPH code generated 2.8 million particles to fill our two-dimensional numerical basin $(60 \times 2.4 \mathrm{~m})$; see Fig. 3. The simulation was run a parallel computing platform (CUDA enabled GPU). Our goal was to predict 


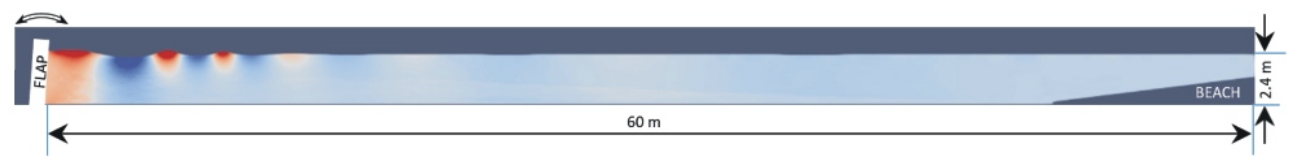

Fig. 3. The DualSPHysics simulation in a meshless 2D domain with 2.8 million particles

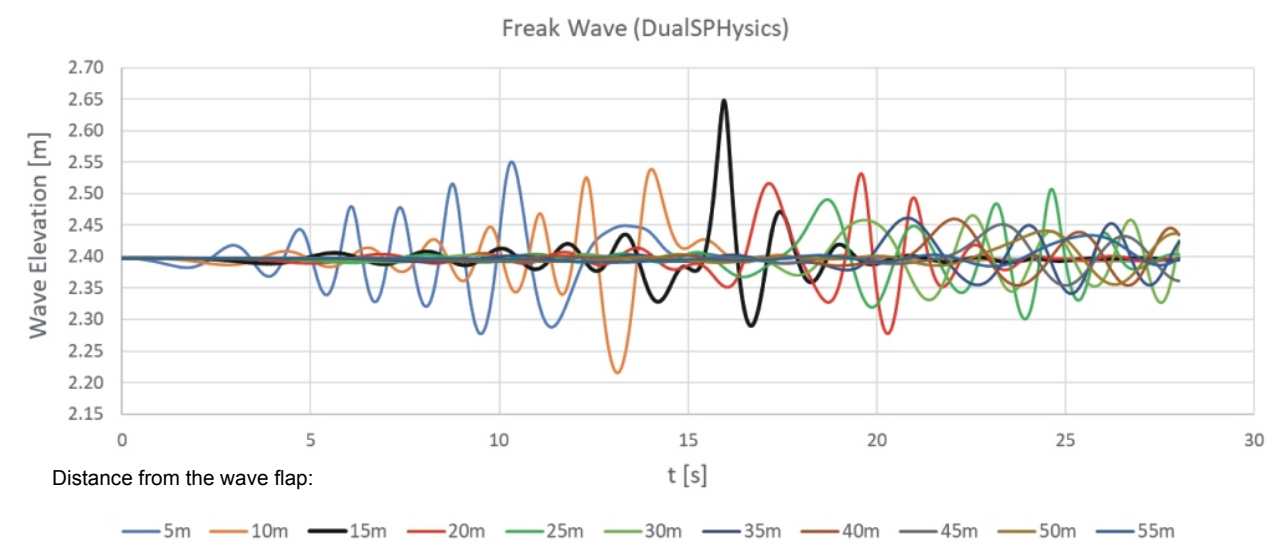

Fig. 4. The numerical results showing $0.25 \mathrm{~m}$ maximum wave amplitude, $15 \mathrm{~m}$ from the wave flap

the location and the amplitude of the maximum wave crest. The results are presented in Fig. 4.

\section{EXPERIMENTAL VALIDATION}

The wave flap motion used in the SPH simulation is duplicated by the physical wave flap in the BSHC seakeeping basin. The wave amplitudes are measured at the four most critical locations predicted by DualSPHysics, situated from $10 \mathrm{~m}$ to $25 \mathrm{~m}$ from the wave maker. The experimental results and the simulations are in good agreement, with an amplitude peak within $2 \%$ of the predicted $0.25 \mathrm{~m}$; see Fig. 5 and Table 1. Figure 6 shows a photo of the maximum crest in the basin.

Before we to run the freak wave described above, a control signal with 1.5 times reduced amplitudes was sent to the wave maker to ensure that the DualSPHysics predictions are reliable and the maximum wave would not be spilt outside the basin walls. Again, the numerical predictions are in good agreement with the experiment, which shows that we can simulate well different 

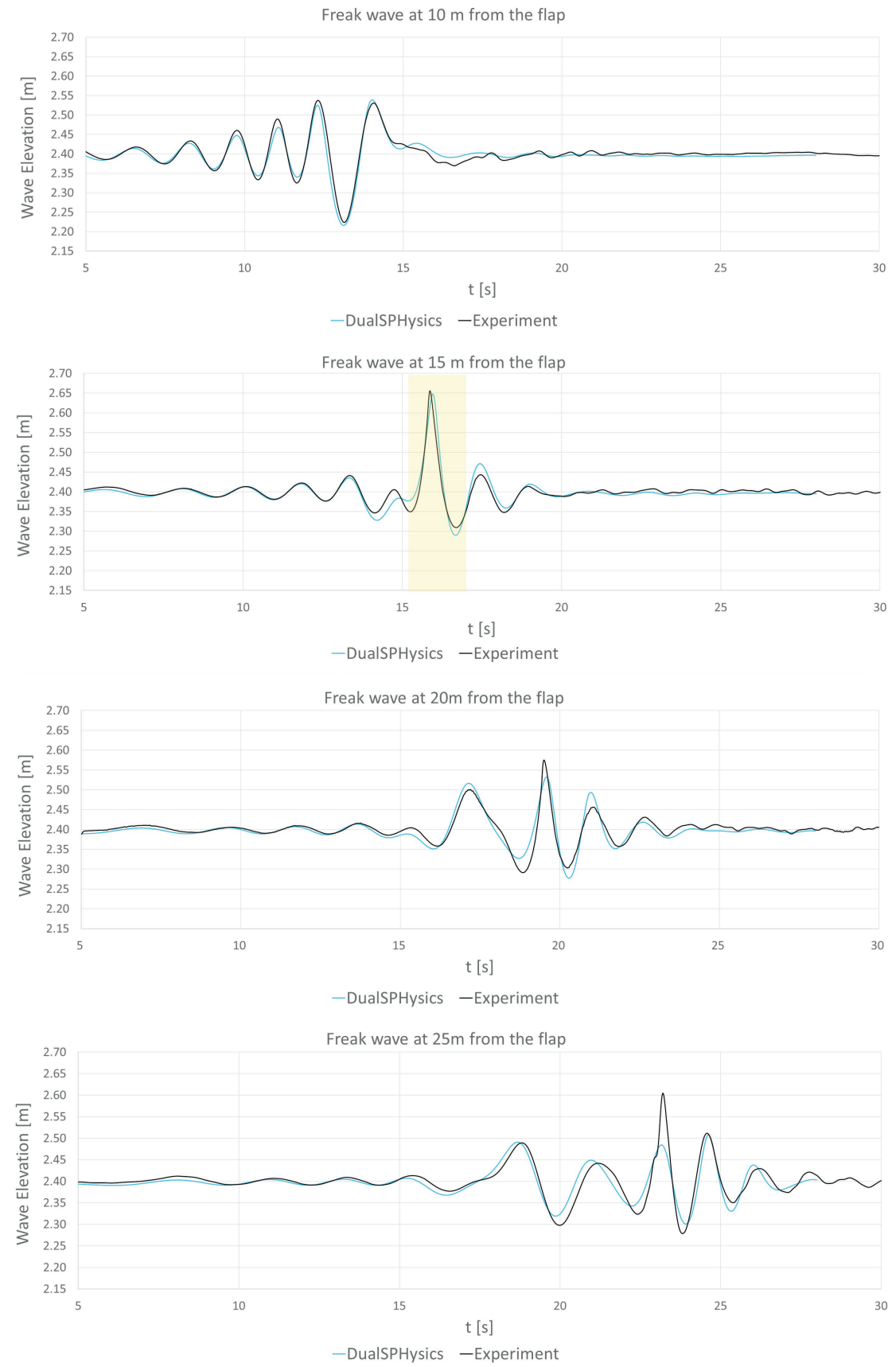

Fig. 5. Comparison between the measurements and the numerical predictions at four locations 
Table 1. Statistics from the measured freak wave in the basin

\begin{tabular}{|c|c|c|c|c|c|c|c|c|c|}
\hline Channel & Units & Mean & Max & Min & StD & Hs & Skewness & Kurtosis & Hmax/Hs \\
\hline WP_10m & $\mathrm{m}$ & 0.00 & 0.14 & -0.18 & 0.03 & 0.13 & -0.36 & 14.19 & 2.5 \\
\hline WP_15m & $\mathrm{m}$ & 0.00 & $\mathbf{0 . 2 6}$ & -0.09 & 0.03 & 0.10 & 4.28 & 43.06 & $\mathbf{3 . 4}$ \\
\hline WP_20m & $\mathrm{m}$ & 0.00 & 0.18 & -0.11 & 0.03 & 0.10 & 0.64 & 14.35 & 2.7 \\
\hline WP_25m & $\mathrm{m}$ & 0.00 & 0.20 & -0.12 & 0.03 & 0.12 & 0.69 & 12.33 & 2.6 \\
\hline
\end{tabular}

Table 2. Statistics from the measured reduced wave in the basin

\begin{tabular}{|c|c|c|c|c|c|c|c|c|c|}
\hline Channel & Units & Mean & Max & Min & StD & Hs & Skewness & Kurtosis & Hmax/Hs \\
\hline WP_10m & $\mathrm{m}$ & 0.00 & 0.10 & -0.12 & 0.02 & 0.09 & -0.18 & 14.48 & 2.5 \\
\hline WP_15m & $\mathrm{m}$ & 0.00 & $\mathbf{0 . 1 4}$ & -0.09 & 0.02 & 0.08 & 1.82 & 23.08 & $\mathbf{3 . 0}$ \\
\hline WP_20m & $\mathrm{m}$ & 0.00 & 0.09 & -0.09 & 0.02 & 0.08 & 0.19 & 9.69 & 2.2 \\
\hline WP_25m & $\mathrm{m}$ & 0.00 & 0.10 & -0.08 & 0.02 & 0.09 & 0.25 & 7.44 & 2.0 \\
\hline
\end{tabular}

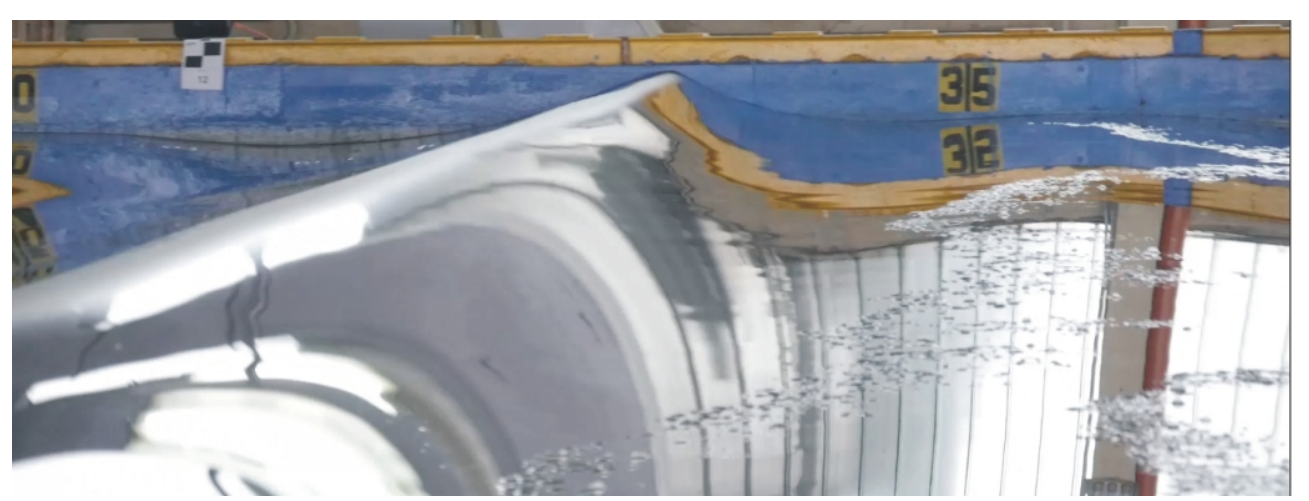

Fig. 6. The maximum wave crest in the basin

degrees of nonlinearity (wave steepness); see Fig. 7 and Table 2.

After validating the numerical model, we compared the freak wave generation by the wave flap (with angular motion) to a wave made by a wave piston (with horizontal linear motion). The wave piston produces a $73 \%$ higher crest maximum when applying the same linear displacement (as at the top of the wave flap) to the piston.

The freak wave generation exercise helped us reasoning why these waves occur rarely. The constructive interference leading to an abnormally high peak requires a unique ratio of the crest periods to the timing of the crest appearance, which is not commonly seen in irregular deep-water waves. The JONSWAP waves, for example (Fig. 8), have similar wave periods within their 


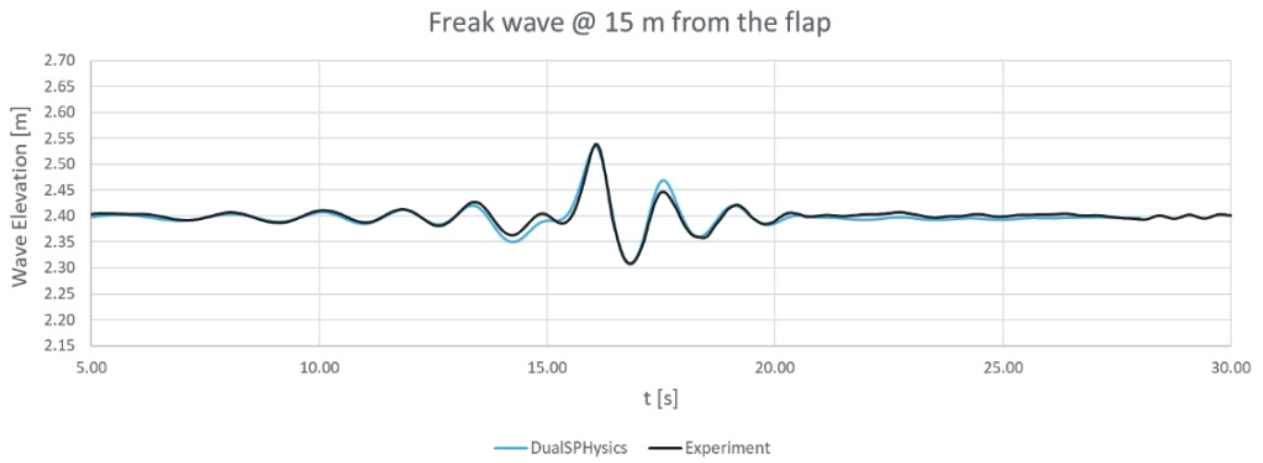

Fig. 7. Comparison between simulations and experiment for the reduced wave

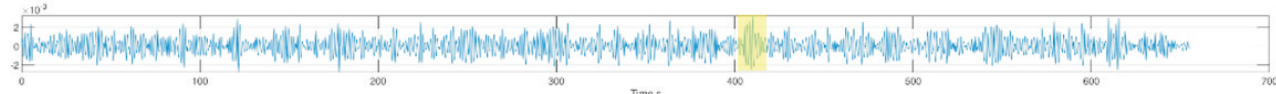

Fig. 8. Irregular wave time series sample based on JONSWAP spectrum

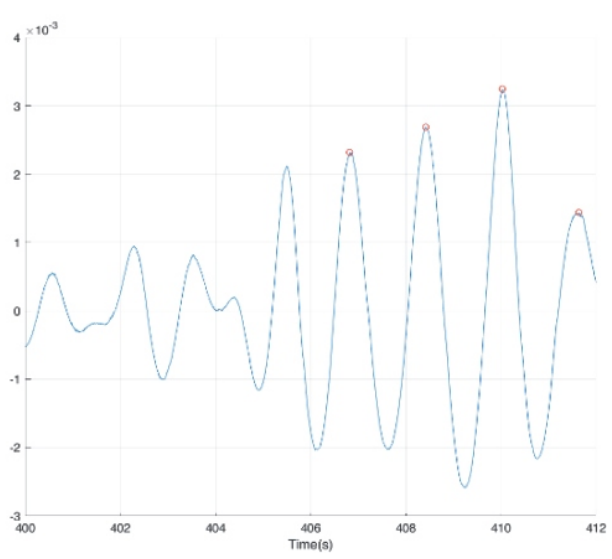

Fig. 9. The highlighted section from Fig. 8

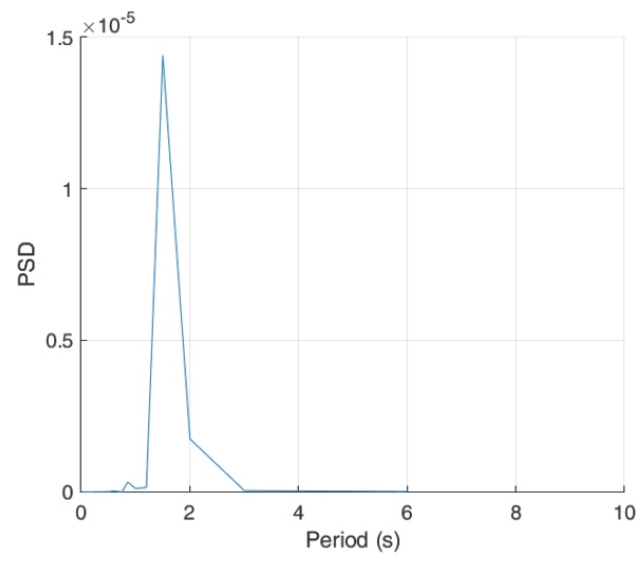

Fig. 10. The spectrum corresponding to Fig. 9

largest groups (Fig. 9). The periods of the waves leading to an abnormal peak are spread much farther, which is evident when comparing the spectra shown in Figs 10 and 11.

It would have been easier to generate a freak wave if we were allowed to send individual waves with different periods one at a time, in a discrete way. This approach, however, does not work because individual waves dissipate 

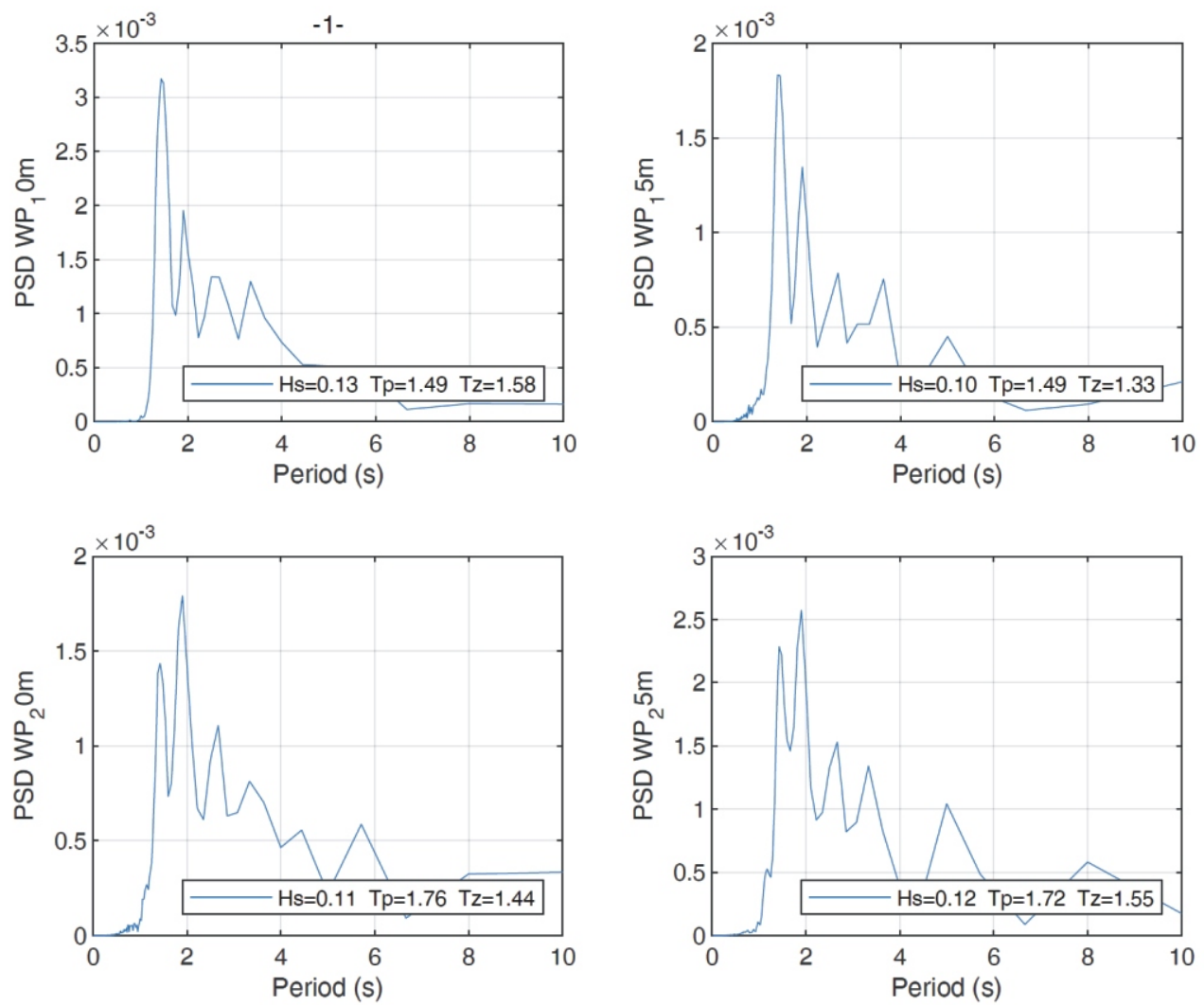

Fig. 11. Wave spectra from the four measured locations in the basin

fast, so we had to smoothly increase the period and the amplitudes (Fig. 1) to avoid early breaking and dissipation. Once we are constrained to the gradual increase of the wave parameters, the sequence has to be tuned uniquely, in an artificial way, to produce the freak crest. The resulting freak wave spectrum does not resemble the common spectra of the wind waves.

\section{CONCLUSION}

Freak waves were successfully created numerically and experimentally. The meshless DualSPHysics simulations and the wave basin tests are in good agreement regardless of the nonlinearity and the steepness of the maximum wave crests. The very tight constraints involved in the freak wave generation point to why such waves are so rarely encountered offshore. 


\section{ACKNOWLEDGEMENTS}

This work was partially supported by the Bulgarian Ministry of Education and Science under the National Research Program "Young Scientists and Postdoctoral Students" approved by DCM \# 577/17.08.2018.

\section{REFERENCES}

[1] T. Adcock And P. TaYlor, Reports on Progress in Physics (2014) 77 105901, DOI: 10.1088/0034-4885/77/10/105901.

[2] F. G. Clauss and M. Klein, Ocean Engineering (2011) 38 (14-15) 1624-1639, ISSN 0029-8018, DOI: 10.1016/j.oceaneng.2011.07.022.

[3] A. B. Buhanobskin And L. I. Lopatuhin, Fundamental and Applied Hydrophysics (2011) 4 (4) 5-17.

[4] C. Kharif, E. Pelinovsky, and A. Slunyaev, Rogue Waves in the Ocean, Springer-Verlag, Berlin, Heidelberg (2009), ISBN 978-3-540-88419-4.

[5] V. M. Pashin and N. N. Rakhmanin, Scientific, Technological and Industrial Journal, Sudostroenie (2005) 5 9-16, ISSN 0039-4580

[6] M. Onorato, D. Proment, et al., PLOS (2013) 8 (2), DOI: 10.1371/journal.pone.0054629.

[7] J. Luxmoore, et al., in: Proceedings of the HYDRALAB IV Joint User Meeting, Lisbon, 2014, pp. 1-5.

[8] J. Hennig And F. VAn WalRee, The $14^{\text {th }}$ International Ship Stability Workshop, ISSW, 2014, pp. 210-215.

[9] J. Hennig And E. C. Schmittner, in: Proceedings of the ASME $31^{\text {st }}$ International Conference on Ocean, Offshore and Arctic Engineering OMAE 2012-83150, pp. $79-86$.

[10] B. Groves and N. Abdussamie, Journal of Ocean Engineering and Science (2019) 4 (2), DOI: 10.1016/j.joes.2019.02.001.

[11] J. J. Monaghan, Annual Rev. Astron. Astrophys. (1992) 30 543-574, DOI: 10.1146/annurev.aa.30.090192.002551.

[12] P. Ropero-Guralda, et al., Renewable Energy (2020) 162 1763-1776, ISSN 0960-1481, DOI: 10.1016/j.renene.2020.10.012.

[13] H. Wendland, Adv. Comput. Math. (1995) 4 389-396, DOI: $10.1007 / \mathrm{BF} 02123482$.

Received April 05, 2021 